\title{
Tratamiento de mordida abierta anterior causada por hábito de deglución atípica
}

\author{
Treatment of anterior open bite caused by atypical swallowing habit \\ Heber Misael Ríos Ruiz (ib ${ }^{1 a}$ Ana Cecilia Lavado Torres ${ }^{16}$
}

\begin{abstract}
RESUMEN
El tratamiento de la mordida abierta anterior es un reto para el ortodoncista debido a la dificultad e inestabilidad para su corrección. En este artículo se presenta el caso de un paciente varón, de 16 años y 4 meses de edad, que acude con el padre al Centro Odontológico de la Facultad de Odontología de la Universidad de San Martín de Porres para ser atendido en la Especialidad de Ortodoncia. De acuerdo al análisis clinico y examenes auxiliares se concluye que presenta: Maloclusión clase I con mordida abierta anterior. El paciente recibio tratamiento correctivo para el hábito de deglusion atipica, para lo cual se utilizó una rejilla palatina. También se realizaron exodoncias de las primeras premolares superiores e inferiores. Al final del tratamiento se observaron cambios faciales y dentales. Se logró el cierre de la mordida abierta, una adecuada alineacion de los dientes, se redujo la protrusion excesiva de los iincisivos tanto superiores como inferiores y una correcta oclusión. En el aspecto facial se observó una notable mejora en el perfil facial, disminucion de la incompetencia labial y la retrusión del labio superior e inferior.
\end{abstract}

Palabras clave: Mordida abierta; Hábito; Deglución atípica; Exodoncias de premolares. (Fuente: DeCS BIREME)

\begin{abstract}
The treatment of anterior open bite represents a challenge for orthodontist due the difficulty and instability of correction. This article presents the case of a 16-year-old male patient who attended the Dental Center of the San Martín de Porres University School of Dentistry - Specialty of Orthodontics, who presented class 1 malocclusion with anterior open bite. The patient received corrective treatment for the atypical swallowing habit, for which a palatal grid was used. Extractions of the upper and lower first premolars were also performed. At the end of the treatment, facial and dental changes were observed. The closure of the open bite was achieved, adequate alignment of the teeth, excessive protrusion of the upper and lower incisors and a correct occlusion were reduced. In the facial aspect, a notable improvement in the facial profile was observed, as well as a decrease in labial incopetence and the retraction of the upper and lower lip.
\end{abstract}

Key words: Open bite; Habit; Atypical swallowing; Premolar extractions. (Source: MeSH NLM)

\footnotetext{
${ }^{1}$ Universidad de San Martin de Porres, Facultad de odontología. Lima, Perú.

${ }^{a}$ Cirujano dentista

${ }^{b}$ Especialista en Ortodoncia y Ortopedia Maxilar.
}

Este es un artículo de acceso abierto distribuido bajo la licencia Creative Commons Atribución 4.0 Internacional (CC BY 4.0) https://creativecommons.org/licenses/by/4.0/deed.es

Correspondencia:

Heber Misael Ríos Ruiz

Dirección: Av. San Luis 1265, San Luis. Lima, Perú.

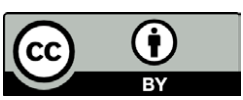

Correo electrónico: drmisael86@gmail.com

Citar como: Ríos Ruiz HM, Lavado Torres A. Tratamiento de mordida abierta anterior causada por hábito de deglución atípica. KIRU. 2021; 18(3):160-171. https://doi.org/10.24265/kiru.2021.v18n3.05 


\section{INTRODUCCIÓN}

La mordida abierta anterior puede ser definida como la presencia de un traspase vertical negativo existente entre los bordes incisales de los dientes antero superiores e inferiores, convirtiéndose en una discrepancia en sentido vertical ${ }^{(1)}$.

Además de comprometer la zona de los incisivos, algunas veces se extiende a los caninos y con menor frecuencia a los premolares y molares. Esta maloclusión, generalmente altera el perfil y acarrea deficiencias en el aspecto estético del paciente, dificultando la aprehensión y corte de los alimentos, además de perjudicar determinados fonemas, exponiendo al paciente a situaciones desagradables en su ambiente. ${ }^{(2)}$.

La mordida abierta anterior puede ser dividida en dos categorías:

- Mordida abierta dental o adquirida, en la cual el patrón esquelético vertical no influye y se originan por alteraciones en la erupción de los incisivos y su extensión se presenta de canino a canino. El 75$80 \%$ de ellas pueden mejorar de manera espontánea, sin requerir ningún tratamiento.

- Mordida Abierta esquelética, es la manifestación tardía de una maloclusión no tratada, y aunque muestra a veces signos similares, son generalmente detectados a partir de la pubertad. Es una displasia ósea constitucional en la cual influyen, sin duda, factores ambientales (lengua, respiración oral patrón hereditario) ${ }^{(3)}$.

El desarrollo de la mordida abierta anterior es el resultado de la interacción de ciertos factores causales; como son las desarmonías esqueléticas en las que se pre senta un crecimiento vertical excesivo, falta de balance muscular, hábitos como succión digital, función anormal de la lengua, obstrucción de las vías aéreas o tratamientos iatrogénicos ${ }^{(4)}$. La mordida abierta es enormemente estudiada por la ortodoncia por ser la maloclusión más difícil de tratar y mantener.

En la literatura se observa una variedad amplia de tipos de tratamientos para la corrección de esta maloclusión, desde la fase debe realizarse un diagnóstico minucioso de cada una de las maloclusiones y sus deformidades esqueléticas, planificando cada tratamiento de manera individual, en vez de adaptar un tipo de maloclusión a un tipo de tratamiento. Así mismo, es necesaria la identificación del factor etiológico siendo esa la clave para un buen plan de tratamiento ${ }^{(5)}$.

Las diferentes modalidades de tratamiento para la mordida abierta fueron estudiadas, con el propósito de disminuir la recidiva, incluyendo aparatos miofuncionales, aparatología fija, spurx linguales, elásticos intermaxilares, alambres, intrusión de molares, extrusión de los dientes antero superiores, cirugía ortognática, exodoncia de premolares, glosectomía parcial o terapia orofacial miofuncional ${ }^{(6)}$. Así, es importante resaltar que la fase de elección para el tratamiento de la mordida abierta debe iniciarse durante la dentición decidua y mixta, cuando se puede evitar un compromiso esquelético; ya que si se interviene a edad mayor la estabilidad y éxito del tratamiento se verán afectados ${ }^{(5,7)}$.

Diversos investigadores concluyen que la posición y actividad de la lengua son las razones por las cuales es difícil lograr estabilidad a largo plazo, encontrando que la terapia orofacial miofuncional combinada con ortodoncia reduce significativamente la posibilidad de recidiva ${ }^{(4,8)}$.

La rejilla palatina fue descrita como el mejor aparato para la corrección de la mordida abierta anterior causada por hábito de deglución atípica. Este aparato es utilizado en el arco superior y puede ser fijo o removible, dependiendo del grado de colaboración del paciente ${ }^{(9)}$. Cozza avaló la efectividad de la rejilla palatina, en un grupo de pacientes en crecimiento con hábitos de deglución atípica y mordidas abiertas esqueléticas y dentarias. Estos pacientes fueron comparados con un grupo control con relaciones verticales semejantes. Los resultados mostraron que el tratamiento con rejilla palatina corrigió la cantidad de mordida abierta anterior $(3.6 \mathrm{~mm}$ más que el grupo control) ${ }^{(10)}$.

Durante la dentición permanente, la decisión de realizar o no exodoncias como auxiliar en el tratamiento ortodóntico, muchas veces causa dudas en los ortodoncistas; sin embargo, la extracción de premolares, es una de las opciones terapéuticas más frecuentes 
para el tratamiento de la mordida abierta anterior ya que ayuda a lograr una compensación en la hiperdivergencia de las bases oseas de ambos maxilares, mediante la mesialización del sector posterior y la consecuente rotación anterior de la mandíbula ${ }^{(11)}$.

Según Sarver y Weissman, se debe evaluar lo siguiente:

- Incisivos superiores e inferiores protruidos o proclinados.

- Exposición gingival de la sonrisa ausente o estrecha.

- Patrón craneofacial normal.

- Exposición del incisivo superior en reposo no mayor de 2 o $3 \mathrm{~mm}^{(12)}$.

\section{Reporte de caso}

Paciente masculino de 16 años y 4 meses de edad, en ABEG, asimétrico, hiperdivergente, dolicofacial, perfil convexo, en dentición permanente con patrón esquelético clase II presenta: Maloclusión clase I por discrepancia alveolodentaria con mordida abierta anterior (Figura1).
Al examen introral se observa una dentición permanente, relaciones molares clase I bilateral, relaciones caninas clase II, línea media dentaria superior(LMDS) desviada 2 $\mathrm{mm}$ a la izquierda con respecto a la línea media facial (LMF), línea media dentaria inferior(LMDI) desviada $2 \mathrm{~mm}$ a la derecha con respecto a la línea media dentaria superior(LMDS), overjet de $7 \mathrm{~mm}$, overbite $5 \mathrm{~mm}$, curva de Spee $1 \mathrm{~mm}$. (Figura 2).

La radiografia panorámica muestra estructuras óseas (cornetes, senos maxilares) de características normales. Ligera desviación del tabique nasal hacia al lado izquierdo y 28 piezas permanentes erupcionadas. Las piezas 18 y 28 en proceso de erupción intraósea y las piezas 38 y 48 en proceso de erupción extraósea. Presencia de osteoesclerosis periapical debajo de la pieza 44 (Figura 3 A y B).

Se planificó un tratamiento correctivo para el hábito de deglución atípica, mediante el uso de una rejilla palatina; además de extracciones de piezas $1.4,2.4,3.4,4.4$, para lograr el correcto restablecimiento de la estética, la función, y la estabilidad biológica
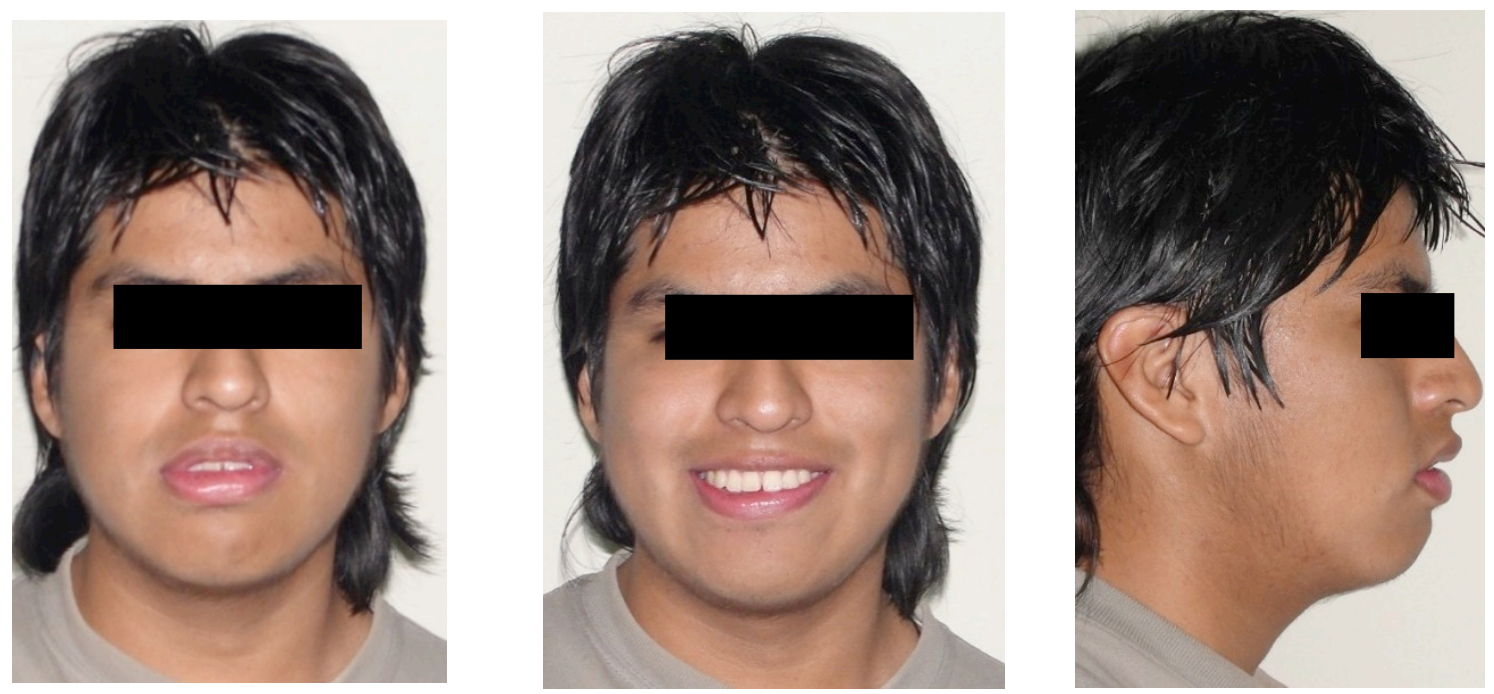

Figura 1. Fotografías extraorales Iniciales 

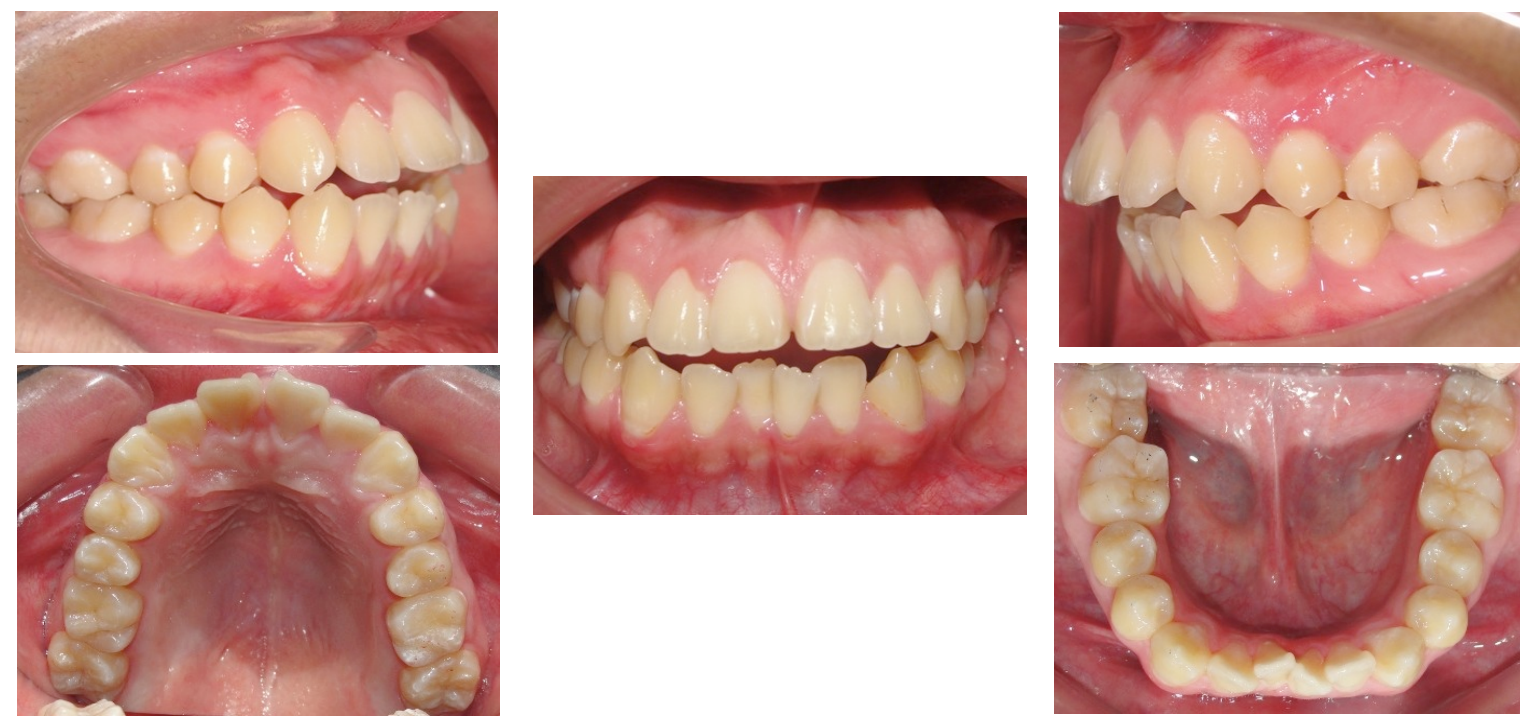

Figura 2. Fotografías intraorales Iniciales

tomando en consideración las indicaciones para seleccionar esta alternativa como son: una discrepancia alveolo dentaria mayor entre el tamaño los dientes y su base ósea, inclinaciones dentarias excesivas producto del hábito instaurado. Se inició el tratamiento con el uso de un Arco Transpalatino ( ATP) más una rejilla palatina soldada a bandas con tubos triples, y un arco lingual, soldado a bandas con tubos dobles convertibles para un control de anclaje moderado (.
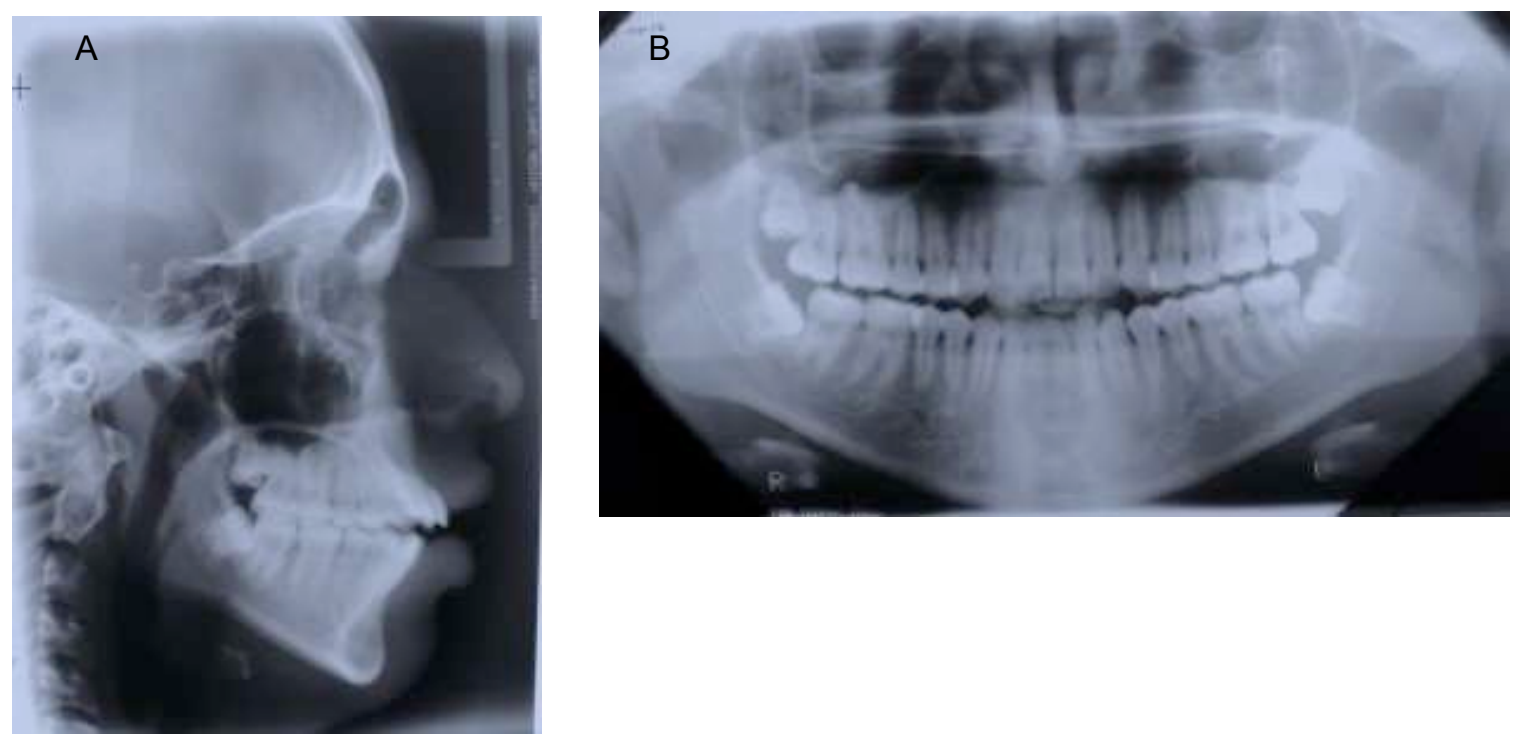

Figura 3 A y B. Fotografías de la radiografía cefalométrica y panorámica inicial. 

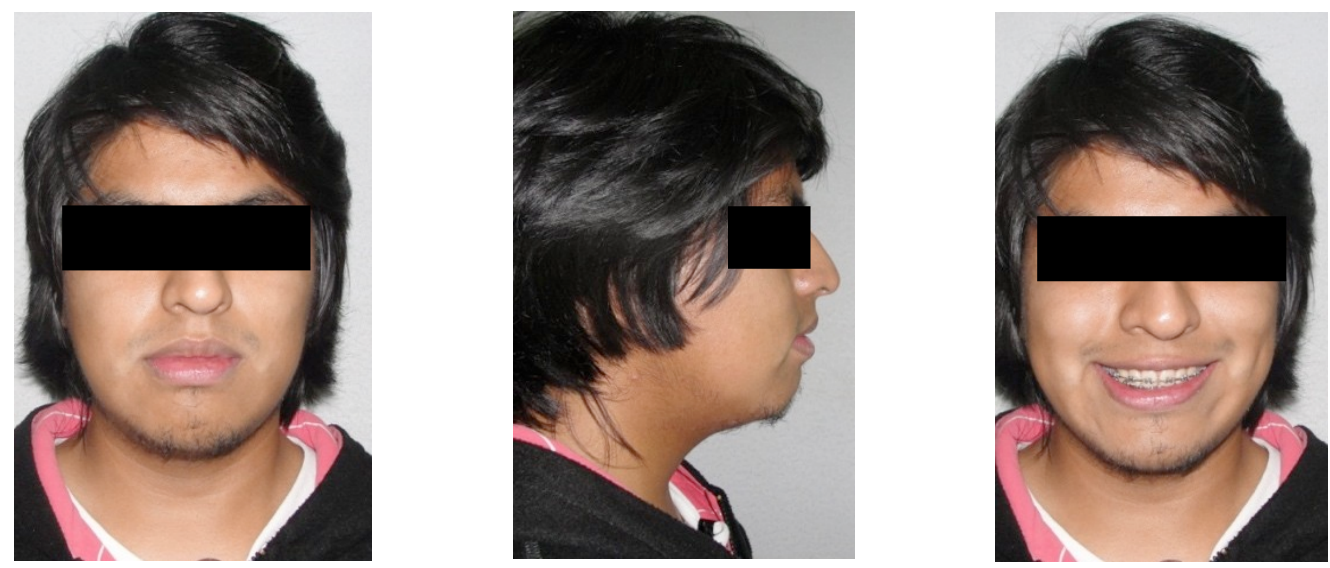

Figura 4. Fotografias Extraorales Intermedias

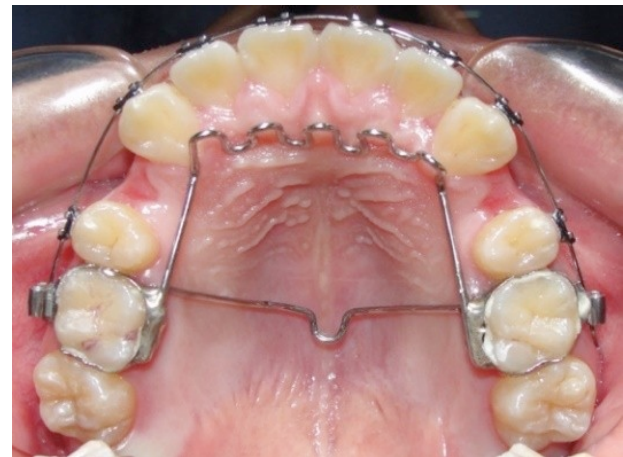

Figura 5. Fotografía Arco Transpalatino más rejilla palatina durante la fase dealineamiento y nivelación
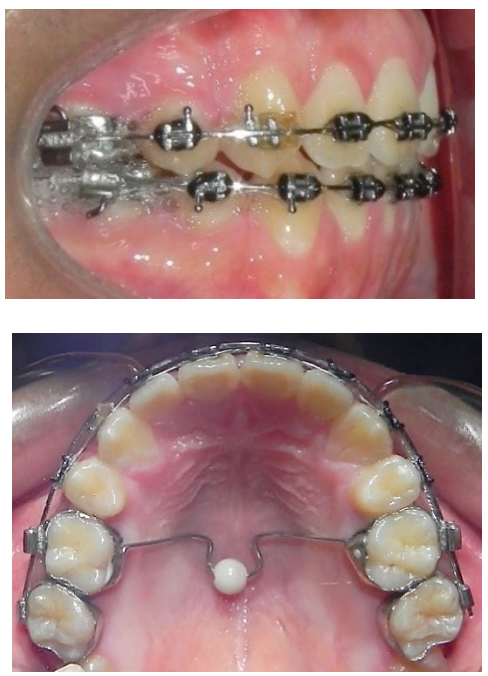

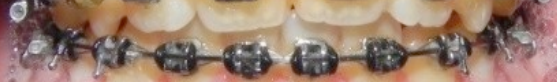

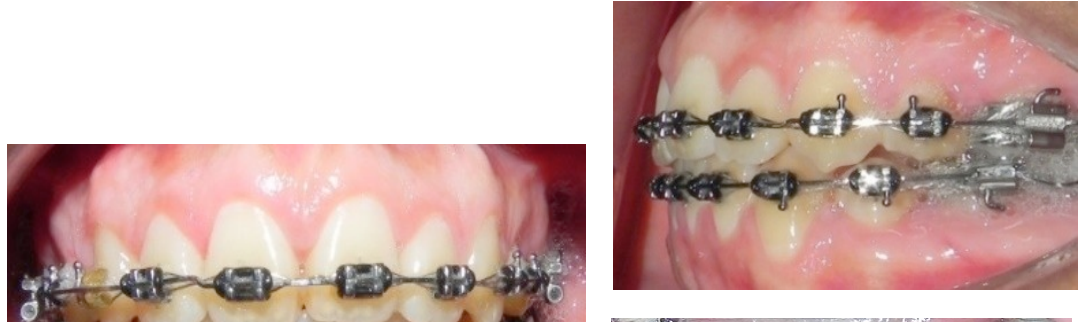

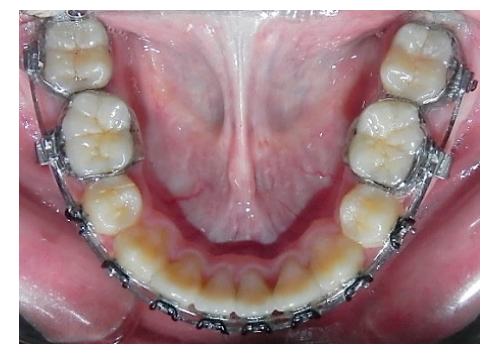

Figura 6. Fotografias intraorales intermedias 
Una vez conseguido el cierre de espacios, se tomaron las fotografías, radiografía panorámica y Cefalométrica intermedias; se evaluó el paralelismo radicular y se procedió a realizar el reposicionamiento de brackets de las piezas 1.2, 2.2, y 3.1(Figura 6 A y B).
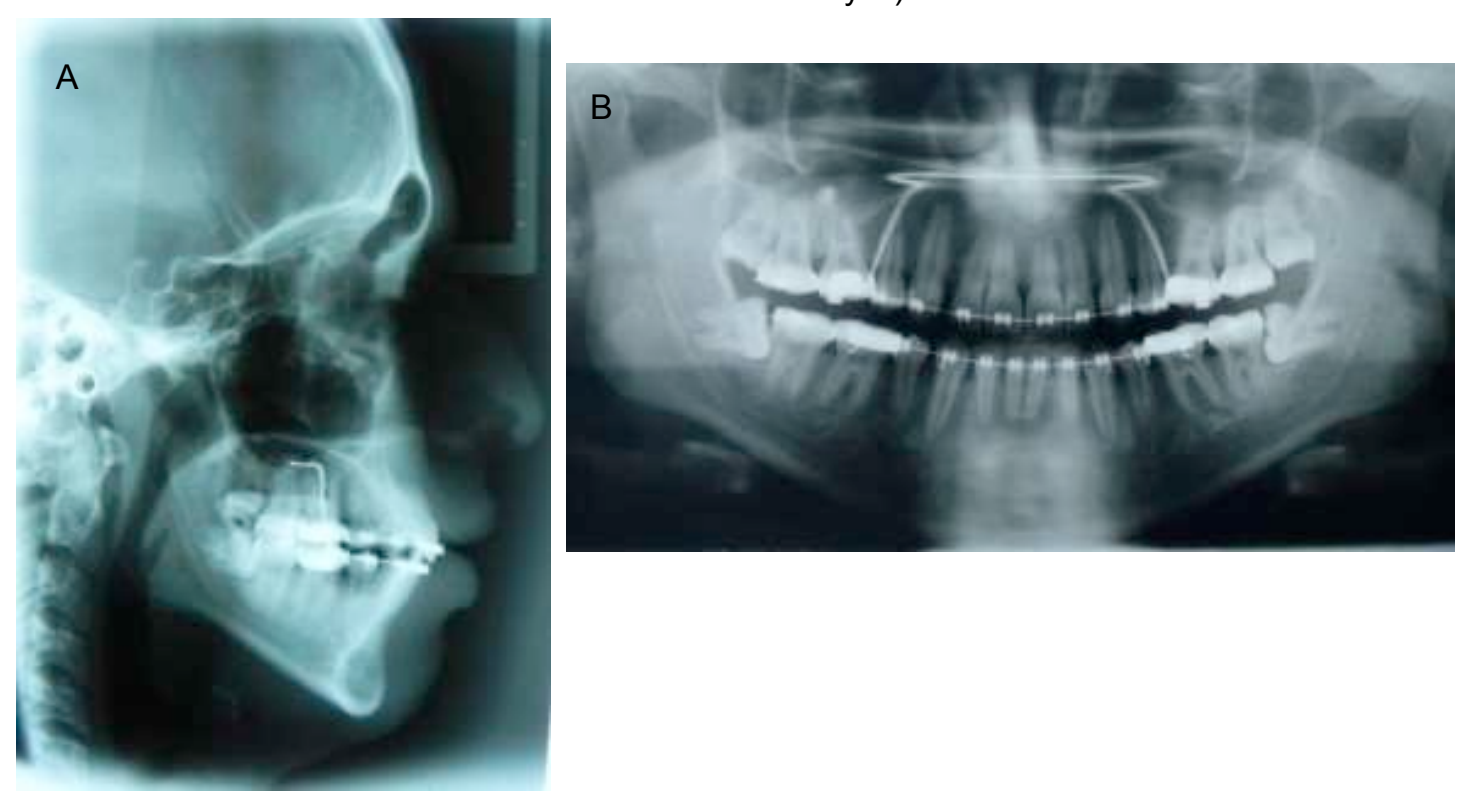

Figura 7 A y B. Fotografías de la radiografia cefalométrica y panorámica intermedia

Luego de culminada la fase de alineamiento y nivelación y cierre de espacios se retiró la rejilla palatina y se cambió por un Arco transpalatino más Perla de Tucat (Figura 7).
Se observó una gran mejora en el perfil facial del paciente, debido al cierre de espacios y de la mordida abierta.

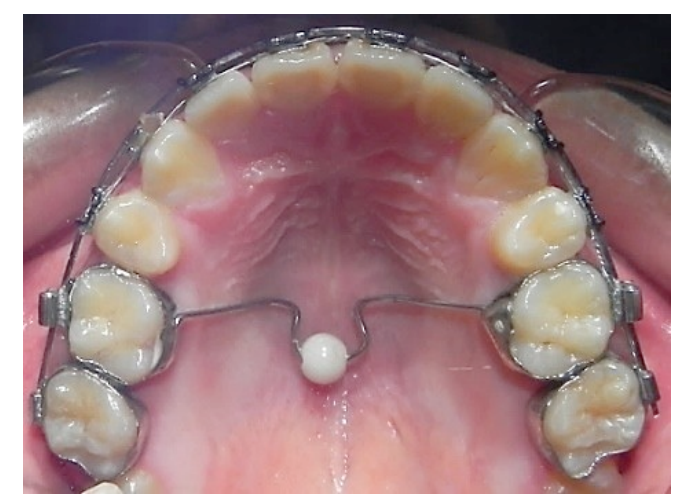

Figura 8. Arco transpalatino más Perla de Tucat 
En las fotografías faciales finales (Figura 8), se observa una sonrisa agradable, con dientes correctamente alineados, competentencialabial y se mantuvo la sonrisa asimétrica, con buena exposición dentaria, y correctos corredores bucales.

En la fotografía de perfil se observa una mejora en el perfil facial y en la biprotrusión labial. En las fotografías intraorales se observa, que se logró centrar las líneas medias, relaciones caninas y molares de clase I, se mejoró la forma de las arcadas. Además, se logró corregir la excesiva proclinación y protrusión de los incisivos superiores e inferiores. Se consiguió un overjet y overbite adecuado, la oclusión en general se dejó estable, y se logró una intercuspidación ideal de los dientes sin interferencias oclusales.
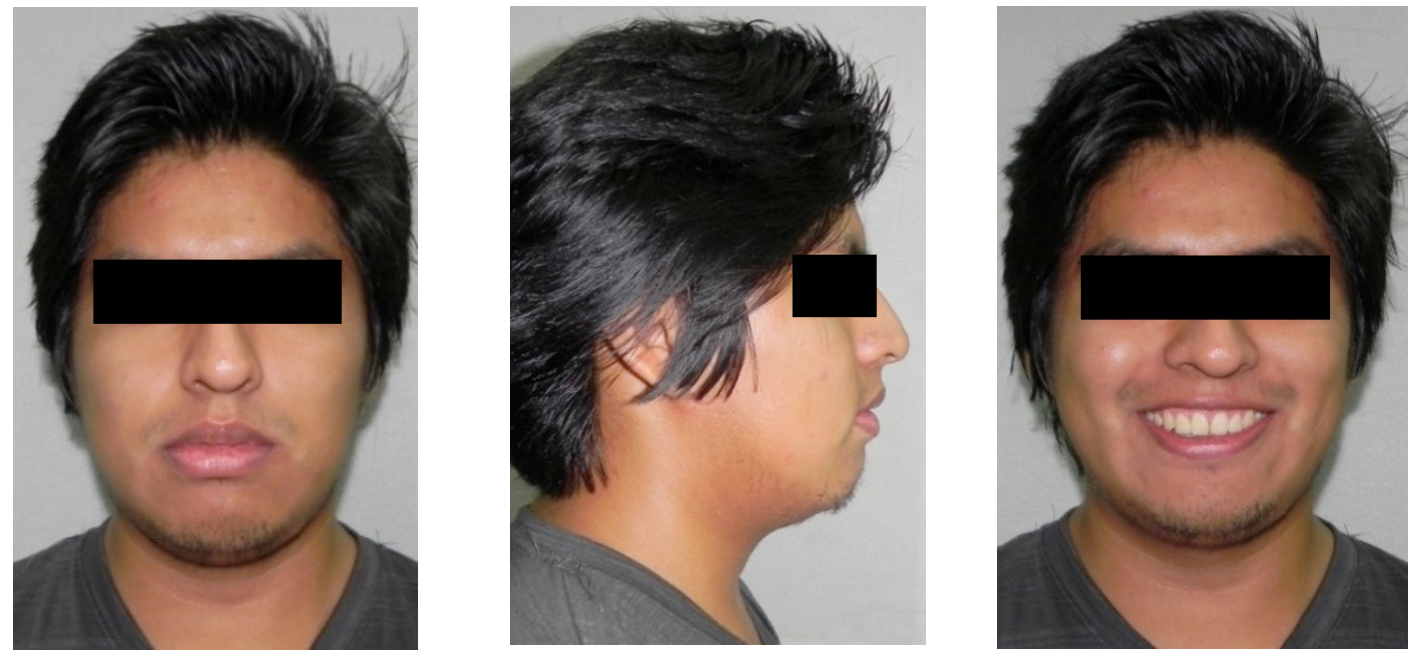

Figura 9. Fotografías extraorales Finales
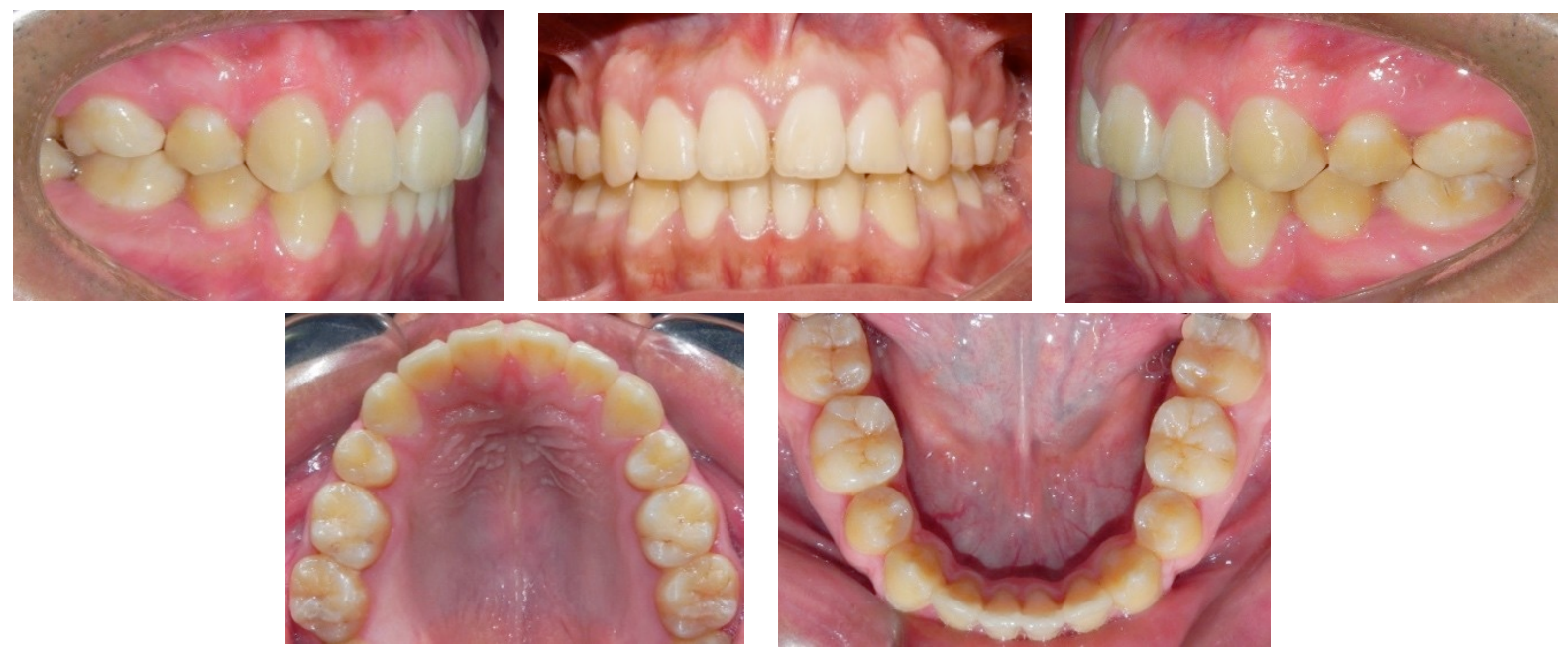

Figura 10. Fotografias finales Intraorales 
En la radiografía panorámica final se observa la presencia de buen paralelismo radicular, se le indico las exodoncias de las terceras molares superiores e inferiores (Figura 10 A).En la radiografia cefalometrica lateral final observamos una correcta inclinación de los incisivos superiores e inferiores con un perfil armonioso (Figura 10 b).
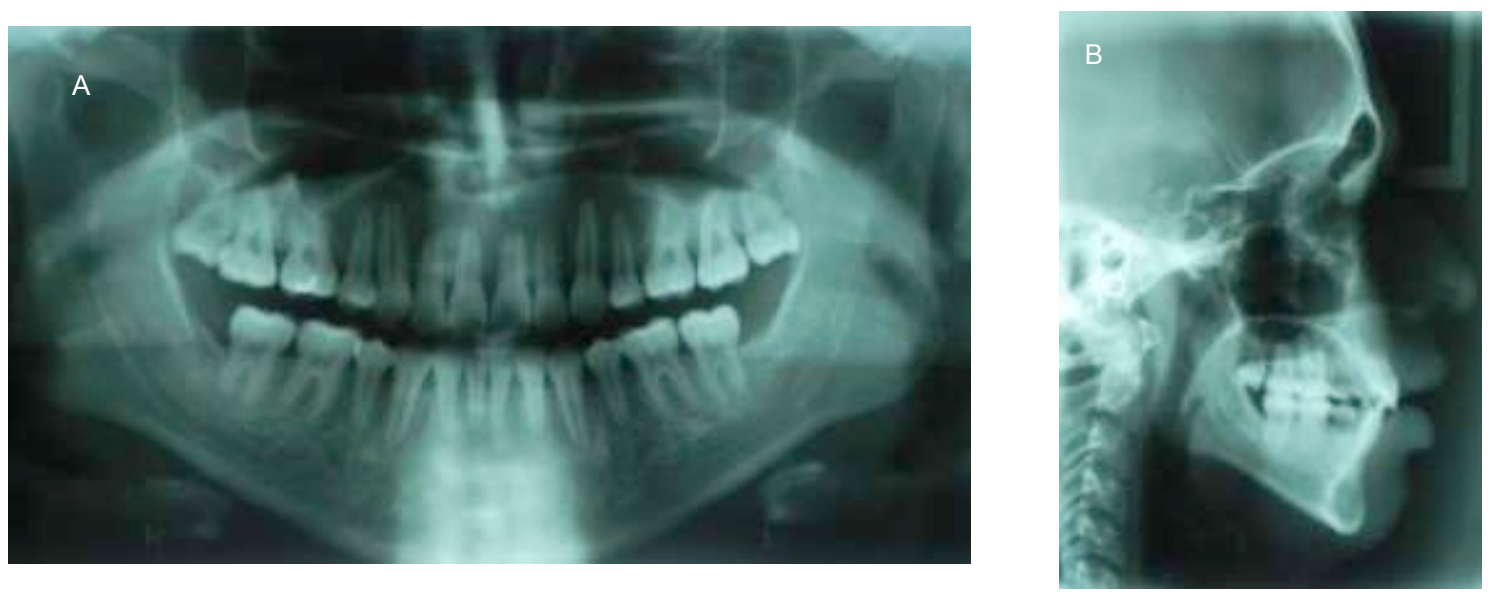

Figura 11 A y B. Radiografías panorámica y cefalometrica finales
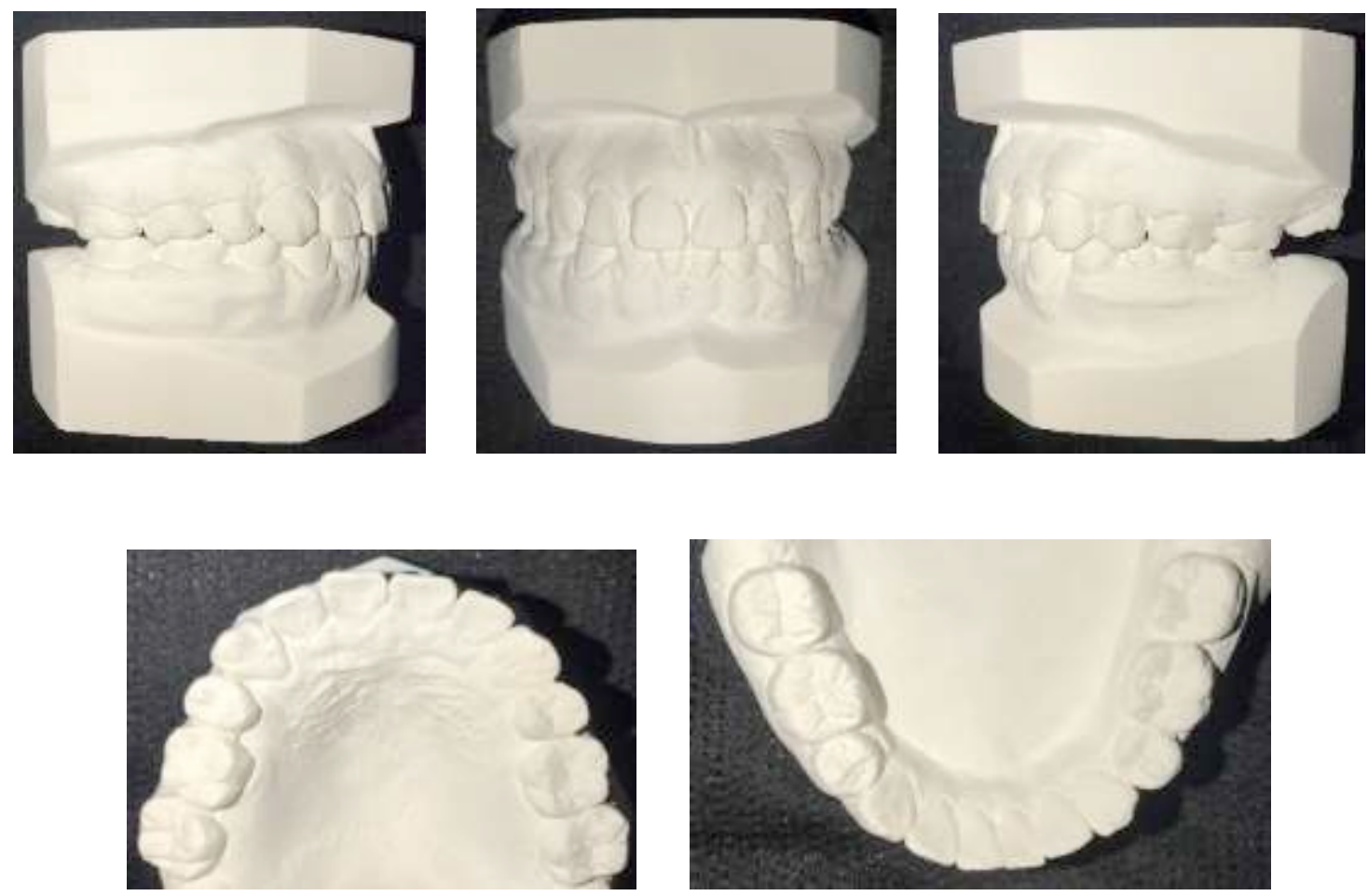

Figura 12. Modelos de estudio 


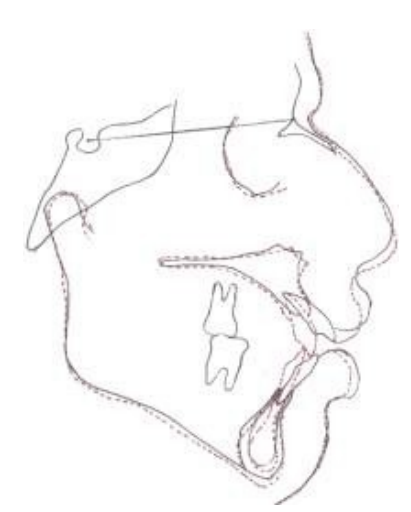

A

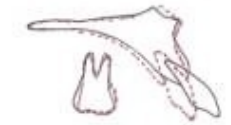

C

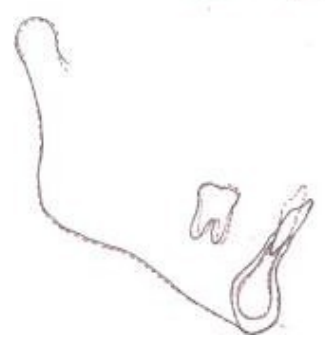

El tratamiento se inició el 27/09/2010 y culminó el 16/06/2013. Al evaluar los cambios se observó: (Fig. 29)

- Desplazamiento hacia adelante y hacia abajo del maxilar superior e inferior producto del crecimiento.

- Retroclinación y retrusión de los incisivos superiores

- Retroclinación y retrusión de los incisivos inferiores.

- Se perdió anclaje en molar superior debido al cierre de espacios.

- Se perdió anclaje en la molar inferior debido al uso de elásticos intermaxilares.

- Hubo una retrusion del labio superior e inferior, mejorando el perfil facial.

Tabla 1. Cuadro de evaluación Cefalométrica. Criterios esqueléticos

\begin{tabular}{|c|c|c|c|c|c|}
\hline & FECHA & $27 / 09 / 10$ & $17 / 10 / 12$ & $14 / 06 / 13$ \\
\hline & & EDAD & 16 años & 18 años & 19 años \\
\hline \multicolumn{6}{|c|}{ CRITERIOS ESQUELÉTICOS NL } \\
\hline \multicolumn{2}{|l|}{ SNA } & $82^{\circ}$ & $86^{\circ}$ & $85^{\circ}$ & $84^{\circ}$ \\
\hline \multicolumn{2}{|l|}{ SNB } & $80^{\circ}$ & $81^{\circ}$ & $80^{\circ}$ & $80^{\circ}$ \\
\hline \multicolumn{2}{|l|}{ ANB } & $2^{\circ}$ & $5^{\circ}$ & $5^{\circ}$ & $4^{\circ}$ \\
\hline \multicolumn{2}{|l|}{ EJEY } & $59.4^{\circ}$ & $60^{\circ}$ & $60^{\circ}$ & $59^{\circ}$ \\
\hline \multicolumn{2}{|l|}{ GO -.-GN A SN } & $32^{\circ}$ & $37^{\circ}$ & $28^{\circ}$ & $35^{\circ}$ \\
\hline \multicolumn{2}{|l|}{ FMA } & $25^{\circ}$ & $28^{\circ}$ & $20^{\circ}$ & $28^{\circ}$ \\
\hline \multicolumn{2}{|c|}{ ENA - ENP A FH } & $4^{\circ}$ & $5^{\circ}$ & $4^{\circ}$ & $4^{\circ}$ \\
\hline \multicolumn{2}{|c|}{ NA -- PG A FH } & $87.8^{\circ}$ & $88^{\circ}$ & $89^{\circ}$ & $89^{\circ}$ \\
\hline \multicolumn{2}{|l|}{ SND } & $76^{\circ}$ & $78^{\circ}$ & $77^{\circ}$ & $77^{\circ}$ \\
\hline \multicolumn{6}{|c|}{ VIAS AEREAS NL } \\
\hline SUPERIOR & \multicolumn{2}{|l|}{$15-20 \mathrm{~mm}$} & $16 \mathrm{~mm}$ & $16 \mathrm{~mm}$ & $17 \mathrm{~mm}$ \\
\hline INFERIOR & \multicolumn{2}{|l|}{$11 \cdots 14 \mathrm{~mm}$} & $12 \mathrm{~mm}$ & $13 \mathrm{~mm}$ & $14 \mathrm{~mm}$ \\
\hline
\end{tabular}


Tabla 2. Cuadro de evaluación Cefalométrica . Criterios dentales

\begin{tabular}{|c|c|c|c|c|c|}
\hline & FECHA & $27 / 09 / 10$ & $17 / 10 / 12$ & $14 / 06 / 13$ \\
\hline & & EDAD & 16 años & 18 años & 19 años \\
\hline \multicolumn{2}{|c|}{ CRITERIOS DENTALES } & $\overline{\mathrm{NL}}$ & & & \\
\hline \multicolumn{2}{|c|}{1 MX. A NA GRADOS } & $22^{\circ}+-2$ & $39^{\circ}$ & $22^{\circ}$ & $21^{\circ}$ \\
\hline \multicolumn{2}{|c|}{$1 \mathrm{MX} . \mathrm{A} N \mathrm{NAmm}$} & $4+-2 \mathrm{~mm}$ & $11 \mathrm{~mm}$ & $4 \mathrm{~mm}$ & $3 \mathrm{~mm}$ \\
\hline \multicolumn{2}{|c|}{$1 \mathrm{MD}$. A NB GRADOS } & $25^{\circ}+-2$ & $36^{\circ}$ & $23^{\circ}$ & $23^{\circ}$ \\
\hline \multicolumn{2}{|c|}{$1 \mathrm{MD} . \mathrm{A} \mathrm{NB} \mathrm{mm}$} & $4+-2 \mathrm{~mm}$ & $12 \mathrm{~mm}$ & $6 \mathrm{~mm}$ & $5 \mathrm{~mm}$ \\
\hline \multicolumn{2}{|c|}{ ÁNGULO INTERINCISAL } & $135^{\circ}$ & $95^{\circ}$ & $134^{\circ}$ & $132^{\circ}$ \\
\hline \multicolumn{2}{|c|}{$1 \mathrm{MX} . \mathrm{A}$ SN. GRADOS } & $103^{\circ}+5.02^{\circ}$ & $131^{\circ}$ & $107^{\circ}$ & $100^{\circ}$ \\
\hline \multicolumn{2}{|c|}{1 MD. A PM. GRADOS } & $90^{\circ}$ & $101^{\circ}$ & $85^{\circ}$ & $88^{\circ}$ \\
\hline \multicolumn{2}{|c|}{$1 \mathrm{MX} . \mathrm{A}$ PO. Mm } & $2.7 \mathrm{~mm}$ & $14 \mathrm{~mm}$ & $7 \mathrm{~mm}$ & $4 \mathrm{~mm}$ \\
\hline \multicolumn{2}{|c|}{$1 \mathrm{MX}$. A PALATINO } & $116^{\circ}$ & $134^{\circ}$ & $112^{\circ}$ & $108^{\circ}$ \\
\hline \multicolumn{2}{|c|}{ TEJIDOS BLANDOS } & $\mathrm{NL}$ & & & \\
\hline \multicolumn{2}{|c|}{ ÁNGULO NASOLABIAL } & $90^{\circ}$ a $110^{\circ}$ & $92^{\circ}$ & $105^{\circ}$ & $94^{\circ}$ \\
\hline \multicolumn{2}{|c|}{ ÁNGULO MENTOLABIAL } & $120^{\circ}+-10^{\circ}$ & $110^{\circ}$ & $115^{\circ}$ & $112^{\circ}$ \\
\hline \multirow[b]{2}{*}{ LINEA E } & SUPERIOR & (-) $2 \mathrm{~mm}$ & $0 \mathrm{~mm}$ & $-2 \mathrm{~mm}$ & $-2 \mathrm{~mm}$ \\
\hline & INFERIOR & $0 \mathrm{~mm}$ & $+5 \mathrm{~mm}$ & $+3 \mathrm{~mm}$ & $+2 \mathrm{~mm}$ \\
\hline
\end{tabular}

\section{DISCUSIÓN}

Los hábitos orales no fisiológicos presentes en una maloclusión son definidos como interferencias $u$ obstáculos que muchas veces agravan las relaciones interoclusales de pacientes en etapa de crecimiento. Estos transcurre el tiempo, convirtiéndose muchas veces en mordidas abiertas esqueléticas.

La mejor forma de controlar el hábito de deglución atípica en este caso fue la utilización de una rejilla palatina, con la finalidad de limitar el movimiento de fuerza anterior de la lengua y promover a la reeducación de la misma.

HUANG y colaboradores en 1190, realizaron un estudio sobre la estabilidad de la mordida abierta, luego de la terapia con rejilla palatina, para lo cual recolectaron una muestra de 33 pacientes, de los cuales 26 se encontraba en fase de crecimiento y $7 \mathrm{sin}$ crecimiento. Luego de la evaluación Cefalométrica, encontraron que hubo un aumento significativo del overbite para ambos grupos durante el tratamiento. El grupo sin crecimiento también mostró un aumento significante en overbite durante el periodo post tratamiento, el $17.4 \%$ de la muestra en hábitos cuando permanecen por tiempos prolongados sin recibir tratamiento, pueden originar mordidas abiertas que pueden detectarse en edades tempranas o adultas; estas a su vez son más difíciles de corregir conforme

crecimiento y $0 \%$ de la muestra sin crecimiento presentaron recidiva. Sin embargo, todos los pacientes que alcanzaron una overbite positivo durante el tratamiento, mantuvieron un overbite positivo postratamiento. De esta forma, concluyeron que los pacientes que alcanzaron un overbite positivo, tratados con rejilla palatina, tienen una buena oportunidad de mantener esa corrección luego de que el tratamiento ortodóntico sea finalizado. Lo que ocurrió en ambos grupos de pacientes, y la razón para que existiera esa gran estabilidad, se debió a la modificación de la postura lingual ${ }^{(7-13)}$.

Sakura y Subtelny, no tuvieron mucho éxito con la terapia de rejilla palatina, esto se asoció con el tiempo de uso; ya que, fue menor de 6 meses. Se ha informado de que el uso prolongado de un aparato fijo para la corrección de la mordida abierta y la inhibición de los hábitos produce resultados más favorables ${ }^{(14,15)}$. 
Diversas terapias de tratamiento han sido orientadas a aumentar la estabilidad de los resultados que se han obtenido en pacientes con mordida abierta; autores como Shapiro sugieren; en caso de hábito lingual; esperar un periodo de por los menos 1.5 - 2 años antes de prescindir de la terapia; para lograr una excelente función ${ }^{(15,16)}$. A este paciente se realizó exodoncias de primeras premolares superiores e inferiores, con la finalidad de obtener un correcto cierre de la mordida abierta anterior y mejorar la incompetencia labial.

Janson en 2006, comparó la estabilidad de la corrección de la mordida abierta anterior con y sin exodoncias y observó una menor recidiva $(25 \%)$ en los pacientes tratados con exodoncias que sin ellas (38\%), lo cual podría deberse a que cuando se hace un tratamiento sin exodoncias la extrusión de los incisivos es menor ${ }^{(17)}$.

\section{CONCLUSIONES}

- Cuando nos encontramos con un caso de mordida abierta lo más imperativo es buscar el factor etiológico de dicha maloclusión y así corregir tempranamente y evitar su progresión.

- En este caso el tratamiento con rejilla palatina y exodoncias de primeras premolares superiores e inferiores, los cambios a nivel dentario fueron: cierre de la mordida abierta, mejorando la proclinación y protrusión dentaria.

- Los cambios faciales logrados con el tratamiento fueron, disminución de la incompetencia labial; logrando descenso y retrusión del labio superior y retrusión del inferior.

- Como retenedor se usó una placa Hawley con rejilla, para evitar la de recidiva de la mordida abierta.

Contribuciones de autoría: HMRR y ALT participaron en el diagnóstico y tratamiento del paciente, así como en la redacción y aprobación del manuscrito.

Fuente de financiamiento: autofinanciado

Conflictos de interés: los autores declararon no tener conflicto de interés.

\section{REFERENCIAS BIBLIOGRAFICAS:}

1. Ngan P, Fields HW. Open bite: A review of etiology and management.OrthoDent, 1997;19(2):91-8.

2. Clemens C.Prevaléncia de mordida aberta anterior em scolares de Sáo Paulo; Universidade Federal do Rio Grande do Soul:1997.

3. Denny JM, Weiskircher MA, Dorminey JC. Anterior open bite and overjet treated with camouflage therapy. Am J Orthod Dentofacial Orthop. 2007; 131 (5): 670678.

4. Justus R. Correction of anterior open bite with spurs: long term stability. World J Orthod. 2001; 2: 219-231.

5. Kim YH. Anterior open bite and its treatment with multiloop edgewise. Angle Orthod.19867;57:290-321.

6. KUHN, R. Control of anterior vertical dimension and proper selection of extraoral anchorage. Angle Orthodont., v.38, p.340-50, 1968.

7. Huang GJ, Justus R, Kennedy DB, Kokich VG. Stability of anterior open bite treated with crib therapy, Angle Orthod. 1990;60(1):17-26.

8. Smithpeter J, Covell D Jr. Relapse of anterior open bites treated with orthodontic appliances with and without orofacial myofunctional therapy. Am J Orthod Dentofacial Orthop. 2010; 137(5): 605-614.

9. Fränkel R, Fränkel C. A functional approach to treatment of skeletal open bite.Am J Orthod. 1983;84(1):54-68.

10. Cozza P, Baccetti T, Franchi LToffol L,McNamara Jr, Mandibular Changes produced by Functional appliances in clas II Malocclusion: A sistematic Review. AJO. 2008;(5):8

11. De Freitas, $M, R$,;Beltrao, $R$ T.;Janson, G.; Henriques, J, F,; Cancado, R.H Longterm Stability of anterior open bite extracción treatment in the permanent dentition. Am.Orhtod. Dentofacial.Orhtop., St. Louis, v 125, n. 1, p 78-87, jan 2004.

12. Sarver D: M. and Weissman S.M. Non surgical treatment of open bite in nongrowing patients AJODO 1995;105:65159.

13. Shapiro Peter. Stabiliity of open bite treatment. Am J Orthod dentofacial Orthop. 2002;121:566-8.

14. Subtenly JD, Sakuda M: Open bite: diagnosis and treatment. Am J Orthod 50:337-58, 1964.

15. Reitzik M, Barer PG, Wainwright WM, Lim B. The surgical treatment of skeletal anterior open-bite deformities with rigid internal fixation in the mandible. Am J 
Orthod Dentofacial Orthop. 1990;97(1):52-7.

16. Baek MS, Choi YJ, Yu HS, Lee KJ, Kwak J, Park YC. Long-term stability of anterior open-bite treatment by intrusion of maxillary posterior teeth. Am J Orthod Dentofacial Orthop. 2010 Oct;138(4):396. e1-396.e9. doi:

10.1016/j.ajodo.2010.04.023.
18. Janson, G.R. P.; Metaxas, A.; Woodside, D. G. Variations in maxillary and mandibular molar and incisor vertical dimension in 12- year-old subjects with excess, normal, and short lower anterior face height. Amer. J. Orthodont. Dentofac. Orthop., v.106, n.4, p.409- 18, Oct. 1994.

Heber Misael Ríos Ruiz

ORCID iD: 으 https://orcid.org/0000-0002--7390--6503

drmisael86@gmail.com

Ana Lavado Torres

ORCID iD: • • https://orcid.org/0000-0002-4835--7178

anacelat@hotmail.com 